\title{
Macroprolactin: the Role of Serial Measurement Throughout Pregnancy
}

Authors

Affiliations

\author{
M. Livingston ${ }^{1}$, S. Nair ${ }^{2}$, D. Radford ${ }^{2}$, J. Kane ${ }^{3}$, A. H. Heald ${ }^{2,4}$
}

Affiliation addresses are listed at the end of the article

\author{
Key words \\ - macroprolactin \\ - hyperprolactinaemia \\ pregnancy \\ - case
}

\section{Abstract \\ $\nabla$}

Introduction: Prolactin circulates predominantly as a $23 \mathrm{kDa}$ monomer, and a high molecular weight form largely consisting of a complex of prolactin and anti-prolactin IgG autoantibody called macroprolactin. The latter cross-reacts with conventional laboratory assays for prolactin.

We here describe the use of serial macroprolactin measurement to assist in the management of a pregnant lady with pre-conception hyperprolactinaemia in whom a magnetic resonance imaging scan had not been performed.

Case Report: A 24-year old lady was found to have a raised prolactin level of $2332 \mathrm{mu} / \mathrm{L}$ (reference range: $40-530 \mathrm{mu} / \mathrm{L}$ ). Prolactin was checked because of primary infertility. Macroprolactin screening was positive, accounting for $54 \%$ of measured prolactin (estimated monomeric prolactin $=1073 \mathrm{mu} / \mathrm{L}$ ).

received 12.08.2015

first decision 07.10.2015

accepted $\quad 11.11 .2015$

Bibliography

DOI http://dx.doi.org/

10.1055/s-0035-1565236

Published online: 2015

Exp Clin Endocrinol Diabetes

Rep 2015; 3: e17-e20

(C) J. A. Barth Verlag in Georg Thieme Verlag KG Stuttgart · New York ISSN 2196-7407

\section{Correspondence}

\section{Dr. M. Livingston}

Department of Blood Sciences Walsall Manor Hospital Walsall, WS2 9PS

UK

Tel.: + 44/1922/656 483

mark.livingston@nhs.net

License terms

$(\Theta(\odot \Theta \Theta$
As expected, measured prolactin levels rose through pregnancy to $6975 \mathrm{mu} / \mathrm{L}$ at 20 weeks and to $8374 \mathrm{mu} / \mathrm{L}$ at 36 weeks gestation.

\section{Introduction \\ $\nabla$}

Prolactin is an anterior pituitary hormone. It was identified in animals as early as 1933 [1] but only purified in humans in 1972 [2]. In the majority of individuals, prolactin circulates predominantly in the form of a $23 \mathrm{kDa}$ monomer $(80 \%$ of circulating prolactin; the active form), with trace amounts of a 50-60 kDa dimeric species ('big' prolactin; accounts for $10-15 \%$ ) and a highmolecular-weight ( $>150 \mathrm{kDa}$ ) complex of monomeric prolactin and commonly an anti-prolactin IgG autoantibody termed 'big-big' prolactin or macroprolactin, which can be identified on gel filtration chromatography $[3,4]$. As a result of its high molecular mass, macroprolactin is confined to the vascular system in vivo and hence is not
Adjustment for macroprolactin and big prolactin gave estimated monomeric prolactin levels of $2370 \mathrm{mu} / \mathrm{L}$ at 16 weeks (macroprolactin/big prolactin $=66 \%$ ), $2441 \mathrm{mu} / \mathrm{L}$ at 20 weeks (macroprolactin/big prolactin $=65 \%$ ) and $2596 \mathrm{mu} / \mathrm{L}$ at 36 weeks (macroprolactin/big prolactin $=69 \%$ ). Thus, adjusted prolactin fell within the reference range for pregnancy at these points.

Gel filtration chromatography was performed on the 20-week (second trimester) sample and compared with the pre-pregnancy sample. The pattern remained similar but all components were increased in the second trimester sample (including the monomeric, big prolactin and macroprolactin forms).

Conclusion: In this case report, serial measurement of macroprolactin with estimation of monomeric prolactin enhanced clinical management throughout pregnancy, saving unnecessary further investigations and avoiding unnecessary distress for the patient.

bioavailable [5]. Delayed metabolic clearance together with detection by many automated prolactin immunoassay systems [6] leads to pseudohyperprolactinaemia in patients harboring this form of complexed prolactin.

It was 1974 when Rogol and Rosen [7] reported for the first time that big prolactin may be the predominant form of prolactin in the serum of some patients with hyperprolactinaemia, while in 1981 Whittaker et al. [8] observed that fertility was maintained even when circulating levels of macroprolactin were significantly elevated. The symptoms of hyperprolactinaemia that prompt measurement of prolactin are non-specific and may occur coincidentally in patients who present with hyperprolactinaemia due to macroprolactin $[9,10]$. 
Fraser et al. [11] showed that all subjects demonstrated a rise in both monomeric prolactin and macroprolactin during pregnancy, with a consistent tendency for monomeric prolactin to increase to a proportionately greater extent than macroprolactin. The data suggested that plasma concentrations of macroprolactin are much less sensitive to pregnancy than prolactin.

We here describe the use of serial macroprolactin measurement to assist in the management of a pregnant lady with pre-conception hyperprolactinaemia in whom a magnetic resonance imaging (MRI) scan had not been performed.

\section{Case Report \\ $\nabla$ \\ Background}

A 24-year old lady was found to have a raised prolactin level of $2332 \mathrm{mu} / \mathrm{L}$ (reference range: $40-530 \mathrm{mu} / \mathrm{L}$ ). Prolactin was checked because of primary infertility in the context of a normal menstrual cycle of duration 30 days. Thyroid function was normal.

Before MRI imaging of the pituitary gland could be performed, she conceived her first pregnancy.

The lady whose case is described was not taking any medication during the period of monitoring reported here.

\section{Laboratory analyses}

Routine assays

Serum prolactin levels were measured using a 2-site chemiluminescent immunometric assay on the Siemens Immulite 2500 analyser (Siemens Immulite 2500 analyser; Siemens Healthcare Diagnostics, Frimley, Camberley, Surrey, UK).

\section{Polyethylene glycol precipitation}

To estimate the amount of bioactive monomeric prolactin present, specimens were assayed for prolactin after treatment with polyethylene glycol (PEG) 6000 (25\%). PEG precipitation is a method that is simple and inexpensive to perform and is commonly used as a screening test for macroprolactin. Briefly, $300 \mu \mathrm{L}$ serum, mixed with an equal volume of $25 \%$ (weight/vol) PEG

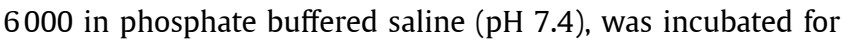
$10 \mathrm{~min}$ at room temperature. After clarification of the suspension by centrifugation for $10 \mathrm{~min}$ at $3000 \mathrm{rpm}$, the monomeric prolactin level in the supernatant was quantified by assay on the Siemens Immulite 2500 . The reproducibility of the PEG precipitation procedure was monitored by inclusion of control sera in each assay.

\section{Gel filtration chromatography}

Gel filtration chromatography of the serum samples was performed at the Department of Clinical Chemistry, Southend Hospital, Westcliff-on-Sea, UK, as previously described [12]. A $60-\mathrm{cm} \times 1.6-\mathrm{cm}$ column of Superdex SD-75 with an AKTA protein purification system (Pharmacia $0.5 \mathrm{~mL}$ of serum was applied to the column and eluted with Tris-buffered saline, at $\mathrm{pH} 7.4$. Fractions $(1.4 \mathrm{~mL})$ were collected and prolactin was measured with the Tosoh AIA 1800 analyser. Macroprolactin, big prolactin, and monomeric prolactin were determined from the area under the peaks.

This is widely viewed as the gold standard reference technique for resolution of the 3 main isoforms of prolactin. It allows direct demonstration of high molecular weight forms of prolactin and quantitative measurement of the monomeric form. This analyti- cal method is slow, labor-intensive, and costly and, therefore, is not suited to routine use. It is commonly used for confirmation and further investigation of equivocal samples by other screening methods (such as PEG precipitation).

\section{Results}

Macroprolactin screening was positive by PEG precipitation at this point, accounting for $54 \%$ of measured prolactin (estimated monomeric prolactin $=1073 \mathrm{mu} / \mathrm{L}$ ). During pregnancy, prolactin levels often increase by 10-20 times (reference values for the $2^{\text {nd }}$ trimester $=2332-6996 \mathrm{miu} / \mathrm{L}$ and for the third trimester $=2904-7886 \mathrm{miu} / \mathrm{L})[13,14]$. In this case, as expected, measured prolactin levels rose through pregnancy to $6971 \mathrm{mu} / \mathrm{L}$ at 16 weeks, $6975 \mathrm{mu} / \mathrm{L}$ at 20 weeks and to $8374 \mathrm{mu} / \mathrm{L}$ at 36 weeks gestation.

However, adjustment for macroprolactin and big prolactin gave estimated monomeric prolactin levels of $2370 \mathrm{mu} / \mathrm{L}$ at 16 weeks ( macroprolactin/big prolactin $=66 \%$ ), $2441 \mathrm{mu} / \mathrm{L}$ at 20 weeks ( macroprolactin/big prolactin $=65 \%$ ) and $2596 \mathrm{mu} / \mathrm{L}$ at 36 weeks ( macroprolactin/big prolactin $=69 \%$ ). Thus, adjusted prolactin fell within the reference range for pregnancy at these points $[13,14]$.

Gel filtration chromatography traces of the 20-week (second trimester) sample compared with the pre-pregnancy sample can be seen in $\bullet$ Fig. 1. The pattern of the different serum isoforms of prolactin remained similar for both samples, but all components were increased in the second trimester sample (including the monomeric, big prolactin and macroprolactin forms). Total prolactin was $8589 \mathrm{mu} / \mathrm{L}$ (41\% macroprolactin, 24\% big prolactin) and the estimated monomeric prolactin was $3006 \mathrm{mu} / \mathrm{L}$, which is within the reference range for this stage of pregnancy.

\section{Clinical course}

Our patient remained well throughout the pregnancy. Visual fields were formally assessed serially thoughout pregnancy with no abnormality identified. Pregnancy went to term with a healthy baby boy being delivered.

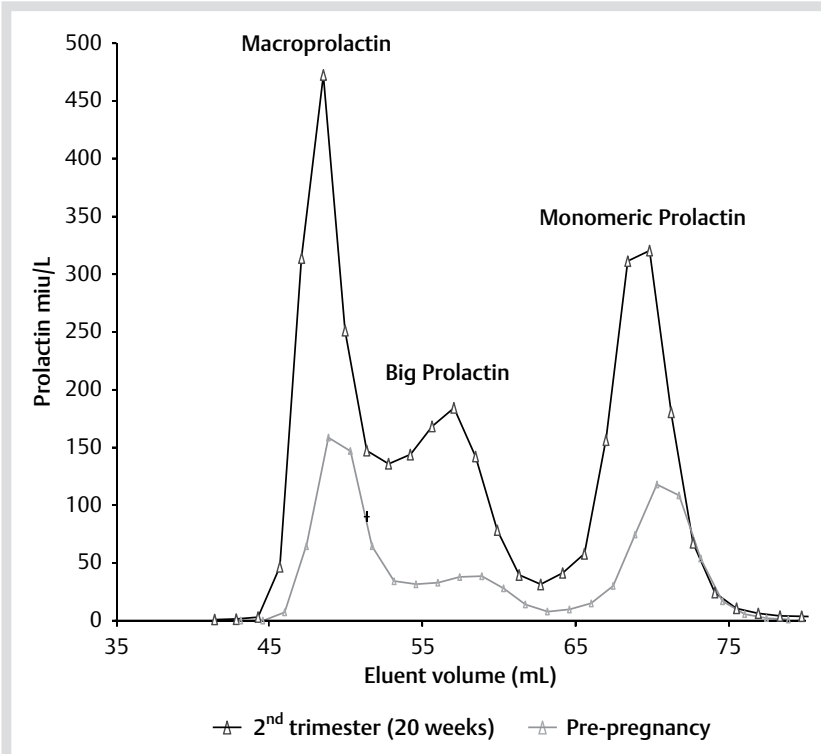

Fig. 1 Gel filtration chromatography of the second trimester sample compared with the pre-pregnancy sample. The pattern is similar but all components are increased in the second trimester sample. 

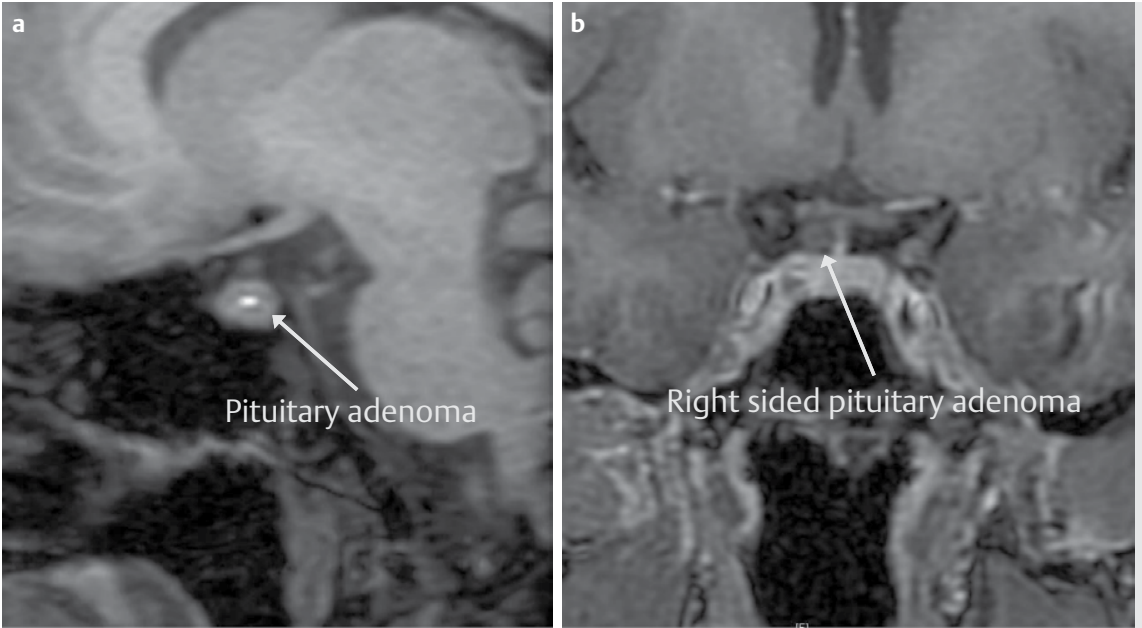

Fig. 2 T2-weighted magnetic resonance imaging scans: a sagittal and $\mathbf{b}$ coronal. Right sided pituitary adenoma, maximum diameter of $5 \mathrm{~mm}$ with evidence of central necrosis/haemorrhage.

An MRI scan of the pituitary was performed post-partum (० Fig. 2). This showed a right sided pituitary adenoma, maximum diameter of $5 \mathrm{~mm}$ with evidence of central necrosis/haemorrhage.

The total prolactin level continued to fall, with an adjusted level after PEG precipitation of macroprolactin being normal at $388 \mathrm{mu} / \mathrm{L}$ 6 months after delivery (reference range: $40-530 \mathrm{mu} / \mathrm{L}$ ).

\section{Discussion}

$\nabla$

Serial measurement of macroprolactin, with estimation of monomeric prolactin, enhanced clinical management throughout pregnancy and avoided unnecessary further investigations. Treatment with Bromocriptine was avoided. Just as importantly, the patient was reassured. Estimated macroprolactin proportions differed as would be expected between those derived from PEG precipitation and those derived from gel filtration chromatography. Gel filtration chromatography indicated that the pattern of the different serum isoforms of prolactin remained similar for both samples, but all components were increased in the second trimester sample.

Our findings contrast with those of Larrea et al. [15] who determined the molecular size heterogeneity of immunoreactive prolactin in the serum from 2 ovulatory hyperprolactinemic women who had large amounts of serum macroprolactin. Serum samples were obtained throughout the menstrual cycle and pregnancy. During the menstrual cycle and the early stages of pregnancy, the serum prolactin in subjects initially was mostly macroprolactin but later in gestation the prolactin composition shifted from the high molecular weight variants to monomeric prolactin. The authors suggested that structural changes in prolactin occur during pregnancy which are probably influenced by the hormonal environment. Furthermore, cord blood obtained at time of birth was shown to contain appreciable quantities of macroprolactin.

In a notable case study by Diver et al. [16], in a pregnant lady, monomeric prolactin increased, but macroprolactin remained the major immunoreactive prolactin component in serum during pregnancy. In a separate study, Hattori [17] concluded that macroprolactinemia was frequently present in the pregnant women studied $(2.9 \%)$ and that different etiologies were involved to form macroprolactin.

As stated in our previous article [18], screening for macroprolactin is a key element of laboratory assessment of hyperprolactinaemia. Laboratory reports should include the adjusted monomeric prolactin level instead of just 'macroprolactin positive' to assist clinical decision making and to avoid unnecessary further investigations. This is particularly important in cases such as this one where tracking of macroprolactin and adjusted monomeric prolactin is being carried out.

Pascoe-Lira et al. [19] studied the presence of anti-prolactin antibodies in the sera of 209 healthy women at different stages of pregnancy. PEG precipitation revealed macroprolactinaemia in 8 of 209 women (3.8\%) and anti-prolactin antibodies were found in 5 of the 8 women. Thus, there is some evidence that macroprolactinaemia may be more prevalent during pregnancy than in the non-pregnant state.

An important clinical point made here is that we avoided unnecessary MRI scans in this lady's pregnancy. Failure to appreciate that macroprolactinaemia during pregnancy leads to unusually high levels of prolactin has led to diagnostic confusion in the past. At least one case report has described inappropriate investigation, including 2 MRI scans, in a woman who subsequently was found to have macroprolactinaemia [9].

The data presented here is further evidence to support the reporting of 'adjusted' monomeric prolactin to aid decision making in the management of patients with hyperprolactinaemia, with application of gel filtration chromatography where appropriate.

\section{Conclusions \\ $\nabla$}

In the case of the pregnant lady reported here, serial measurement of macroprolactin, with estimation of monomeric prolactin, greatly enhanced clinical management throughout pregnancy and saved unnecessary further investigations, avoiding untoward distress for the patient. Screening for macroprolactin is now a key element of laboratory assessment of hyperprolactinaemia. In cases where measured prolactin is significantly raised, determination of macroprolactin status may alter the course of management.

\section{Funding \\ $\nabla$}

No external funding supported this study.

\section{Ethical Approval}

Informed consent obtained. 


\section{Contributors}

$\nabla$

ML provided scientific advice and helped with the literature review and drafting of the manuscript. AHH supervised the research and helped draft the manuscript. DR, JK and SN were involved in data collection and contributed to the writing of the paper. All authors approved the final version of the manuscript.

\section{Guarantor}

$\nabla$

\section{$\mathrm{AHH}$}

Conflict of interest: None to declare.

\section{Affiliations}

${ }^{1}$ Department of Blood Sciences, Walsall Manor Hospital, Walsall, United Kingdom

${ }^{2}$ Department of Diabetes and Endocrinology, Leighton Hospital, Crewe, United Kingdom

${ }^{3}$ Department of Clinical Biochemistry, Salford Royal Hospitals NHS Trust, Salford, United Kingdom

${ }^{4}$ The School of Medicine and Manchester Academic Health Sciences Centre, University of Manchester, Manchester, United Kingdom

\section{References}

1 Riddle 0 , Bates R, Dyshorn S. The preparation, identification and assay of prolactin - a hormone of the anterior pituitary. Am J Physiol 1933; 105: 191-216

2 Hwang P, Guyda H, Friesen H. Purification of human prolactin. J Biol Chem 1972; 247: 1955-1958

3 Suh HK, Frantz AG. Size heterogeneity of human prolactin in plasma and pituitary extracts. J Clin Endocrinol Metab 1974; 39: 928-935

4 Schiettecatte J, De Schepper J, Velkeniers B, Smitz J, Van Steirteghem A. Rapid detection of macroprolactin in the form of prolactin-immunoglobulin $\mathrm{G}$ complexes by immunoprecipitation with anti-human IgG-agarose. Clin Chem Lab Med 2001; 39: 1244-1248

5 Leite V, Cosby H, Sobrinho LG, Fresnoza MA, Santos MA, Friesen HG. Characterization of big, big prolactin in patients with hyperprolactinaemia. Clin Endocrinol 1992; 37: 365-372

6 Cavaco B, Prazeres S, Santos MA, Sobrinho LG, Leite V. Hyperprolactinemia due to big big prolactin is differently detected by commercially available immunoassays. J Endocrinol Invest 1999; 22: 203-208
7 Rogol $A D$, Rosen SW. Prolactin of apparent large molecular size: the major immunoactive prolactin component in plasma of a patient with a pituitary tumor. J Clin Endocrinol Metab 1974; 38: 714-717

8 Whittaker PG, Wilcox T, Lind T. Maintained fertility in a patient with hyperprolactinaemia due to big, big prolactin. J Clin Endocrinol Metab 1981; 53: 863-866

9 Gibney J, Smith TP, McKenna TJ. Clinical relevance of macroprolactin. Clin Endocrinol 2005; 62: 633-643

10 Gibney J, Smith P, McKenna TJ. The Impact on Clinical Practice of Routine Screening for Macroprolactin. J Clin Endocrinol Metab 2005; 90: 3927-3932

11 Fraser IS, Lun ZG, Zhou JP, Herington AC, McCarron G, Caterson I. Detailed assessment of big big prolactin in women with hyperprolactinemia and normal ovarian function. J Clin Endocrinol Metab 1989; 69: 585-592

12 Fahie-Wilson MN, Soule SG. Macroprolactinaemia: contribution to hyperprolactinaemia in a district general hospital and evaluation of a screening test based on precipitation with polyethylene glycol. Ann Clin Biochem 1997; 34: 252-258

13 O'Leary P, Boyne P, Flett P, Beilby J, James I. Longitudinal assessment of changes in reproductive hormones during normal pregnancy. Clin Chem 1991; 37: 667-672

14 Abbassi-Ghanavati M, Greer LG, Cunningham FG. Pregnancy and laboratory studies: a reference table for clinicians. Obstet Gynecol 2009; 114: $1326-1331$

15 Larrea F, Escorza A, Valero A, Hernandez L, Cravioto MC, Diaz-Sanchez $V$. Heterogeneity of serum prolactin throughout the menstrual cycle and pregnancy in hyperprolactinemic women with normal ovarian function. J Clin Endocrinol Metab 1989; 68: 982-987

16 Diver MJ, Ewins DL, Worth RC, Bowles S, Ahlquist JA, Fahie-Wilson MN. An unusual form of big, big (macro) prolactin in a pregnant patient. Clin Chem 2001; 47: 346-348

17 Hattori $N$. The frequency of macroprolactinemia in pregnant women and the heterogeneity of its etiologies. J Clin Endocrinol Metab 1996; 81: $586-590$

18 Heald AH, Blantern E, Anderson SG, Radford D, Qureshi Z, Nair S. Quantitative adjustment for macroprolactin is an integral part of laboratory assessment of hyperprolactinaemia. Exp Clin Endocrinol Diabetes 2012; 120: 376-380

19 Pascoe-Lira D, Duran-Reyes G, Contreras-Hernandez I, Manuel-Apolinar $L$, Blanco-Favela F, Leanos-Miranda A. Frequency of macroprolactinemia due to autoantibodies against prolactin in pregnant women. J Clin Endocrinol Metab 2001; 86: 924-929 